\title{
Finding Their Data Voice: Practices and Challenges of Dashboard Users
}

Melanie Tory

Roux Institute at Northeastern University

Lyn Bartram

Simon Fraser University

Brittany Fiore-Gartland

Tableau Software

Anamaria Crisan

Tableau Research

Abstract-Dashboards are the ubiquitous means of data communication within organizations. Yet we have limited understanding of how they factor into data practices in the workplace, particularly for data workers who do not self-identify as professional analysts. We focus on data workers who use dashboards as a primary interface to data, reporting on an interview study that characterizes their data practices and the accompanying barriers to seamless data interaction. While dashboards are typically designed for data consumption, our findings show that dashboard users have far more diverse needs. To capture these activities, we frame data workers' practices as data conversations: conversations with data capture classic analysis (asking and answering data questions), while conversations through and around data involve constructing representations and narratives for sharing and communication. Dashboard users faced substantial barriers in their data conversations: their engagement with data was often intermittent, dependent on experts, and involved an awkward assembly of tools. We challenge the visualization and analytics community to embrace dashboard users as a population and design tools that blend seamlessly into their work contexts.

SELF SERVICE ANALYTICS within organizations has led to an explosion in the spectrum of workers who actively engage with data. This growth has outstripped the ability of organizations to develop approachable analytics solutions and practices. Yet visualization designers have little understanding of data work practices within organizations and consequently an impoverished foundation for building effective support tools.

Particularly on the rise are data workers, defined by Liu et al. [1] as people who need to engage with data periodically and perform data analysis activities, but who do not identify as data professionals. For many data workers, dashboards ${ }^{1}$ serve as their primary portal to data and the material place where they interact with others around data. Recent research [2] highlighted the ubiquity of dashboards as the data platform within organizations. In this article, we define dashboard

\footnotetext{
${ }^{1}$ Following [2], we define dashboards as either visual data representations structured as tiled layouts and/or interactive displays of dynamically updating data for awareness or decision-making.
} 
users as the subset of data workers who use dashboards or data reports as their primary portal to data AND who do not create such automatic data-driven artifacts for others. Dashboard users hold diverse roles in companies, nonprofit organizations, education, and government; for example, market campaign management, product management, operations, and employee success. They comprise a large proportion of data workers and are often overlooked because their data interactions are intermittent and their data work is thought of as simplistic. In this article, we draw a distinction between professionals who produce dashboards and other data-driven artifacts for others (termed here as analysts) and people who use those artifacts (dashboard users).

While numerous studies have characterized the work practices of professional analysts and data scientists (e.g., [3], [4], [5], [6]), the activities of dashboard users are poorly understood. We address this gap through a foundational interview study of dashboard users and their data sensemaking ${ }^{2}$ practices within organizations. In addition to interviewing dashboard users, we also interviewed analysts to gain information about the dashboard users they support and the struggles they observe dashboard users facing. We sought to understand the range and depth of dashboard users' practices and how to support their sensemaking activities. Our results highlight the technological impediments and breakdowns they encounter in trying to get the job done.

Dashboard users differ from analysts in both the tools they use and the skills they bring. Professional analysts are skilled in the craft of interaction with and transformation of data. Their expertise manifests as data knowledge, extensive time to analyze data problems, an ability to identify patterns, and an ability to represent analytics problems in a principled way [6]. In contrast, few dashboard users would consider data analytics to be the primary function of their role. Our results show that despite deep domain expertise, they often have limited analytics skills and may

\footnotetext{
${ }^{2}$ Following Weick [7], we define sensemaking as "an ongoing accomplishment that takes form when people make retrospective sense of the situations in which they find themselves and their creations". At an organizational level, sensemaking goes beyond individual retrospection; it is a social activity in which information is interpreted in the context of the environment. Dialogue and narrative contribute directly to the process of sensemaking.
}

engage with data intermittently. Opportunities to strengthen their analytics expertise or learn new data tools may be limited, even if they recognize the value of those skills. Even those with technical expertise may have limited time to engage deeply with their data.

Our primary distinction between analysts and dashboard users is whether they produce or use dashboards. However, as we will show, dashboard use cannot be characterized as passive "data consumption": dashboard users engage in many of the same cognitive activities as analysts, but often in an awkward ad-hoc way because existing tools have not been designed with their time constraints and skillsets in mind. We observed that dashboard users actively engage in data analysis, transformation, and translation work. They frequently pull data from their dashboards into other tools to manipulate and understand them. They also repackage data into new artifacts to add contextual information, create new meaning, and share stories and explanation with others.

To capture these diverse data practices of dashboard users, we characterize the data work that they do as data conversations. Conversation with data [3], [6] is the iterative interaction between people and data to ask and answer questions (classic analysis work). We further recognize and highlight collaboration and sharing practices involving data, or conversation through and around data, as critical data practices.

This paper makes the following contributions:

1) A foundational study of dashboard users that elucidates work practices, workflow breakdowns, workaround strategies, and their analytics journeys.

2) A characterization of data work as data conversations, based on constructs from communication theory. We use this to frame the practices of dashboard users, but it may have broader applicability.

Dashboard users are under-recognized and under-served for the range and depth of data work that they do [2]. By understanding their work practices and challenges, our research identifies opportunities for future research and better tool support, establishing a foundation to empower this under-served population. 


\section{RELATED WORK}

We first discuss studies of data work practices. We then review theories on communicative practice involving data that underpin our characterization of data work as data conversations.

\section{Understanding data practices}

Many studies have explored practices of professional analysts in enterprise contexts. Kandogan [3] found data analysis was distributed, collaborative, and involved three levels of analytic work: tactical, operational, and strategic. Studies characterizing the tasks involved in analytical work [4], [5], [6] reported activities including data discovery, wrangling, profiling, modeling, reporting, exploring, curation, and so forth. Kandel et al. [4] reported that workflows typically involve multiple analysts who collaborate asynchronously and use various tools in an iterative and nonlinear process. Looking at a broader population of users, Liu et al. [1] examined how data workers generate, manage, and explore alternatives and Boukhelifa et al. [8] explored how they cope with uncertainty. Koesten et al. [9] examined collaboration across roles, identifying needs for conversation around data, documentation about data, and tools for easier version control and customized data access. Common themes emerge from these studies: data work involves using a variety of tools, revisiting and re-using analyses, and collaborating with others across roles. Although a few of these studies included both professionals and nonprofessionals [8], [9], [1], most participants were data experts with strong technical skills. The extent to which these findings apply to dashboard users remains unclear.

Studies that focus primarily on nonprofessional data workers are limited. Convertino et al. [10] and Erete et al. [11] reported that data workers relied heavily on spreadsheets, especially for data preparation tasks such as combining multiple data sources or transforming data to more consumable formats for target audiences. Moreover, 'casual data analysts' were found to devote about $60 \%$ of their time to data preparation and organization [10]. Similarly, Bartram et al. [12] found that "untidy" data practices in spreadsheets offered data workers a tangible and accessible means of thinking with data. A survey of widespread dashboard use [2] surfaced monitoring, documentation, and communication as important tasks in data work, and a study of designers working with data [13] revealed frequent tool switching, non-linear task sequencing, and benefits of manual encoding as a means of exploration. Finally, a recent study of organizational decision makers [14] identified a need to expand visualization tools beyond support for analysis, to include decision making tasks such as scenario generation and trade-off comparison.

The studies above demonstrate that data workers often require a collection of disjointed tools to accomplish their work. Lack of tool integration was a common complaint. Data organization and reshaping imposed substantial effort and potential for "schema drift" [4] and misinterpretation. Documenting and annotating is complicated by tool disparity and does not travel with the data. Spreadsheets were used frequently - not because of superior function, but because of basic capabilities needed to transition smoothly between data shaping, analysis, documentation, presentation, and communication tasks and also because they enabled users to combine structured and unstructured (i.e. textual) data [12], [3], [10], [11]. Notably, some studies reported less use of interactive visualization than we might expect [12], [3]. Contributing factors could include the effort required to integrate visualization tools with functions of data organization and manipulation, along with technological and visual literacy.

Our work extends knowledge of data work practices in organizations by focusing specifically on dashboard users, chosen because dashboards are a predominant means of data circulation in organizations yet these users are not well understood. We drill in depth into the goals, tasks, and workflow breakdowns encountered by dashboard users. Our results reveal that while dashboards have been designed for data consumption, the analytical needs of dashboard users go far beyond simple consumption tasks. As a result, we highlight opportunities to develop new tool support for this population.

\section{Data as Communicative Practice}

Organizational communication theories can explain the material roles that dashboards and other data artifacts play within organizations. Neff et al. [15] characterized three communicative 
roles of documents: documentation, records or archives documenting decisions or history; circulation, ways to connect actors and ideas; and conversation, material site of conversation for thinking with, through, and around the artifact. In practice, documents and other organizational artifacts play multiple roles. For example, the whiteboard often functions as a material site of conversation as teams sketch ideas. Next, a team member might take a photo of the whiteboard with a smart phone because materially a white board does not support the other communicative functions of circulation and documentation. Data and data analytics, just like documents, have textual and material affordances and are embedded within broader communicative processes. Applying this communication role typology to data analytics helps us make sense of people's data work activities and moments of breakdown.

Functioning as documentation, data provide records of decisions and support collaboration by providing a stable ground upon which others may build or reference. Supporting circulation, data artifacts are distributed throughout an organization, extending data access to many actors. Data democratization efforts often emphasize circulation, or bringing data to more people.

It is the role of data as a site of conversation that begins to shape the practices of sensemaking across an organization. Who gets to interpret the data? Who can have a data voice? Who can ask and answer questions with data? Developing the conversational role of data supports the emergence of new ideas, the evolution of practices, and the adaptation of the organization.

Articulating organizational data work practices reveals the dynamic relationship that people have with data as they converse with, around, and through data. Conversation with data involves interactions directly between the user and their data (individual analysis work) [3], [6]. One important construct with respect to conversation with data is the data's materiality, or its tangibility and ability to support direct manipulation. As expressed by Tanweer et al. [16], "The formal materiality of an Excel spreadsheet can be easily grasped and manipulated; one can open the document and scroll through its rows and columns, copy and paste, edit and delete. But when working with 90 terabytes of text or a trillion rows of data...engaging with its formal materiality is a challenge." The ability to interact and probe the materiality of data helps less skilled people make sense of their data [16].

Of course, data work is not only analysis and not only for oneself. We expand the notion of data conversation from an individual's engagement with data to the larger constructs of sensemaking, discourse, and communication. Conversations through data use the data as a medium for conveying information, a story, or a particular view to others. Key here is that people reshape the data and its associated artifacts as a strategy for communication, anticipating the translation necessary for data to "jump contexts" [17]. Conversations around data refer to the ways the data and associated artifacts may support interactions relevant to the group, including discussing data or what isn't represented in the data. Talk around data may generate further questions and insights, lead to actions, or simply support connection among people [18]. These characterizations elaborate the meaning of data conversation beyond just "interacting with data" and more fully capture the complex dynamics of data work practices. Data conversations comprise more than analysis; they are the substrate of decision-making in many workplaces. We employ these notions of conversations with, through, and around data within our framework describing the data activities of dashboard users.

\section{INTERVIEWS}

We conducted 1 hour semi-structured interviews with 20 participants over video conferencing. We focused on one or two specific dashboards or data reports either created or used by the participant. The artifact(s) served as a probe to ground the interviews, similar to prior research (e.g., [4]). After gaining some context about the person's organization and role, we asked them to screenshare or describe the data artifact and tell the story of its use. We inquired about the artifact's contents, who uses it, goals and tasks it supports, why it is important, collaborative activities around the artifact, other tools used along with it, what it is missing, and so on. Interview guides are in supplemental material. This study was approved by the human research ethics board at Simon Fraser University. 


\section{Participants}

We recruited participants through the usertesting.com online user research platform $(n=16)$, personal referrals $(n=3)$, and social media $(n=1)$. We employed a screener survey to limit participation to qualified candidates. We excluded people who did not work with data in an organizational setting, whose data tasks were limited to data entry or single value lookup, or who reported using any nonexistent analytics tool. We continued to add new participants until saturation was reached.

Based on interview responses, we categorized participants as analysts if they described data analytics as a primary function of their role and either built dashboards for others or supported others to work with data. In contrast, dashboard users relied on dashboards curated by others and did not consider analytics a primary function of their job. By this categorization, 7 participants were analysts and 13 were dashboard users. With dashboard users, we discussed their data work directly. With analysts, we focused on the needs, practices, and skills of their dashboard end users.

There were 9 female and 11 male participants from a wide variety of job roles in 18 organizations, mostly for-profit companies ranging from one employee to thousands. Age ranged from 26 to 63 with an average of 39 and 4 unspecified. They used a variety of analytics tools including spreadsheets, Tableau, PowerBI, and in-app dashboards. Very few had programming skills. Only a few used mobile devices; one dashboard user exclusively used mobile. See participant details in Table 1. Participant names are pseudonyms.

\section{Data and Analysis}

We recorded audio, video, and screencapture. Interviews were fully transcribed. We used a modified grounded theory approach [19] to analyze our data, beginning with open coding and constant comparison of transcripts, later organizing codes into categories. We refined the codes as we gathered more data and repeatedly returned to the transcripts to validate or refute our emerging understanding. Analyst and dashboard user interviews were analyzed simultaneously. One researcher did all of the coding, while engaging two others in frequent discussion.

This process allowed us to identify the conver- sational work with, around, and through data that our participants engaged in, and the breakdowns that occurred throughout this work, as important themes. Breakdowns, or "points at which progress is stopped due to a material limitation" [16], were of particular interest. Tanweer et al. demonstrated how breakdowns can serve as "essential sites of knowledge production" [16] in data science work. This led us to more selectively code those situations to explore both the types of conversational work and the sites of breakdown. Our unit of analysis was instances where dashboard users encountered barriers in completing their tasks within their primary analytics tool. For each breakdown, we characterized the high level goal the person was trying to achieve, the lower level task where they encountered a barrier, and the strategy they took to overcome or evade the breakdown. The coding scheme, documented in the tables and figures in the next section, was developed over multiple passes and was also informed by standard visualization task taxonomies and Neff et al.'s [15] typology of communicative roles of documents. Once it stabilized, we performed an exhaustive pass to apply the coding scheme to all interview statements expressing dashboard users' goals or tasks, even where breakdown did not occur. As our interviews with analysts focused on the experience of their dashboard end users, our unit of analysis (i.e. dashboard user goals/tasks/strategies) was employed for both groups of interviewees. We also coded deliberate strategies taken by analysts to help their end users avoid breakdown.

\section{PRACTICES OF DASHBOARD USERS}

We focused on data activities performed by dashboard users, situations in which they encountered breakdown, and their strategies to mitigate breakdown. We also examined the strategies that analysts employed to help their dashboard users avoid breakdown in the first place.

\section{Goals and Tasks When Working With Data}

Examining the data activities of dashboard users, we identified two high level categories of goals (see Table 2 for a detailed description and Figure 1 for frequencies).

Conversation with data strongly echoed typical analysis tasks in visualization. One noticeable 
Table 1. Interview Study Participants (Mgmt = Management, $\mathrm{IC}=$ Individual Contributor)

\begin{tabular}{|c|c|c|c|c|c|c|c|}
\hline Participant & Context & Group & Age & Gender & Org Size & Role & Analytics Tools \\
\hline Lisa & Retail Art Sales & Dashboard user & 26 & F & Small & Small business owner & Square Dashboard; Google Sheets \\
\hline Milo & Website Content Mgmt & Dashboard user & 38 & $\mathrm{M}$ & Small & Small business owner & Google Analytics; Google Sheets \\
\hline Tammy & Retail Arts Sales & Dashboard user & 38 & $\mathrm{~F}$ & Small & Small business owner & Excel, In-app dashboards \\
\hline Matthew & Retail Beverage Sales & Dashboard user & 44 & $\mathrm{M}$ & Large & Senior Mgmt & Old Database Reporting Tool; Excel \\
\hline Amy & Student Educational Assessment & Dashboard user & 38 & F & Large & Mgmt & Power BI dashboards; Excel \\
\hline Dustin & Fitness Memberships & Dashboard user & 63 & $\mathrm{M}$ & Large & Mgmt & Excel; Dashboards \\
\hline Jeff & Corporate Marketing Campaigns & Dashboard user & 57 & $\mathrm{M}$ & Unknown & Senior Mgmt & Excel; Excel-based database report \\
\hline Kayla & Corporate Administration Services & Dashboard user & 31 & $\mathrm{~F}$ & Large & IC (Admin Assistant) & CRM; Bloomberg; Excel; PowerPoint \\
\hline Avril & Social Media Content Mgmt & Dashboard user & 36 & $\mathrm{~F}$ & Small & Mgmt & Tableau Desktop ${ }^{a}$; In-app dashboards \\
\hline Mandy & Corporate Marketing Campaigns & Dashboard user & 41 & $\mathrm{~F}$ & Large & IC (Campaign Mgmt) & Tableau dashboards; Google Sheets \\
\hline Mateo & Online Content and Engagement & Dashboard user & 37 & $\mathrm{M}$ & Small & Mgmt & YouTube Analytics, Google AdWords \\
\hline Miguel & Corporate Talent Acquisition & Dashboard user & 36 & $\mathrm{M}$ & Large & Senior Mgmt & Tableau Mobile dashboards \\
\hline Wade & Software Performance Accuracy & Dashboard user & Unknown & $\mathrm{M}$ & Large & IC (Program Mgmt) & Excel; Tableau dashboards \\
\hline Liz & Healthcare Program Participation & Analyst & 28 & F & Small (Agency) & IC (Consultant) & Excel; Tableau Desktop \\
\hline Brandon & Healthcare Operations Consulting & Analyst & 29 & $\mathrm{M}$ & Small (Agency) & IC (Consultant) & Excel; Tableau Desktop \\
\hline Fred & Supplier and Purchase Order Mgmt & Analyst & 40 & $\mathrm{M}$ & Large & IC (Procurement) & Excel; Tableau Desktop \\
\hline Maria & IT for Supply Chains & Analyst & Unknown & $\mathrm{F}$ & Large & IC (Project Mgmt) & Excel; Tableau Desktop \\
\hline Kevin & Online Finance Customer Experience & Analyst & Unknown & $\mathrm{M}$ & Large & IC (Program Mgmt) & Splunk; Excel \\
\hline Kaleb & Telecom Sales and Marketing & Analyst & Unknown & $\mathrm{M}$ & Large & IC (Dashboard Developer) & Excel; Tableau Desktop \\
\hline Stacey & Analytic Consulting & Analyst & 47 & $\mathrm{~F}$ & Unknown & IC (Consultant) & Excel, SQL \\
\hline
\end{tabular}

${ }^{a}$ Novice use of the Desktop tool, simply to pull data rather than to construct lasting artifacts.

Table 2. Goals of dashboard users. Numbers indicate how many participants reported (breakdown; the goal)

\begin{tabular}{|c|c|c|}
\hline & Goal and description & Example from our interviews \\
\hline \multirow{9}{*}{$\begin{array}{l}\text { Conversation } \\
\text { with data }\end{array}$} & $\begin{array}{l}\text { Summarize: Obtain a summary or overview of } \\
\text { the data }(13 ; 18)\end{array}$ & $\begin{array}{l}\text { "....what's the sales volume in a given period of time, at a given set of } \\
\text { stores, for these products" [Matthew] }\end{array}$ \\
\hline & $\begin{array}{l}\text { Monitor: Stay up to date with key metrics and } \\
\text { performance indicators }(10 ; 18)\end{array}$ & $\begin{array}{l}\text { "I want those live results. I wanna know right away when we're tweeting, } \\
\text { who's seeing it." [Avril] }\end{array}$ \\
\hline & $\begin{array}{l}\text { Explain: Find the underlying cause behind an } \\
\text { observation }(9 ; 14)\end{array}$ & $\begin{array}{l}\text { "If we...notice that we're getting a lot of fails on [one assignment] we'll } \\
\text { dig deeper and find what part of the assignment are they failing." [Amy] }\end{array}$ \\
\hline & $\begin{array}{l}\text { Predict: Predict an outcome under different } \\
\text { scenarios }(4 ; 12)\end{array}$ & $\begin{array}{l}\text { "....say } 10 \text { members a month it's going up. I may project out what are our } \\
\text { revenue's going to be based on the membership fees." [Dustin] }\end{array}$ \\
\hline & $\begin{array}{l}\text { Compare: Compare two or more entities } \\
(5 ; 12)\end{array}$ & $\begin{array}{l}\text { "...this many customers had high speed in December versus this many } \\
\text { people had high speed in January." [Mandy] }\end{array}$ \\
\hline & Lookup: Look up a fact $(9 ; 11)$ & "How many people from [city] bought security in December?" [Mandy] \\
\hline & $\begin{array}{l}\text { Experiment: Observe the effect of an event or } \\
\text { deliberate change }(4 ; 8)\end{array}$ & $\begin{array}{l}\text { ".... we'll put together a [web] page...and then we'll wait for a while and } \\
\text { see what sort of traffic it gets... sort of like a guinea pig type of page." } \\
\text { [Milo] }\end{array}$ \\
\hline & $\begin{array}{l}\text { Find Anomaly: Seek or identify anything that } \\
\text { is out of the ordinary }(2 ; 5)\end{array}$ & $\begin{array}{l}\text { "When you have goose eggs on your report when you're supposed to have } \\
\text { 30. Yeah, that's probably gonna tell you something." [Jeff] }\end{array}$ \\
\hline & $\begin{array}{l}\text { Audit: Record by record inspection and vali- } \\
\text { dation }(2 ; 4)\end{array}$ & $\begin{array}{l}\text { "....they had a sales tax audit...he just needed to be able to line by line } \\
\text { classify." [Stacey] }\end{array}$ \\
\hline \multirow{4}{*}{$\begin{array}{l}\text { Conversation } \\
\text { through and } \\
\text { around data }\end{array}$} & $\begin{array}{l}\text { Discuss Data: Converse about data, insights, } \\
\text { and actions to take }(8 ; 17)\end{array}$ & $\begin{array}{l}\text { "....it's really a jumping off point for questions and for these people to } \\
\text { have a thoughtful conversation with our managing directors" [Kayla] }\end{array}$ \\
\hline & $\begin{array}{l}\text { Circulate: Disseminate among organizational } \\
\text { actors }(12 ; 14)\end{array}$ & $\begin{array}{l}\text { "I'm on a circulation list...Everybody in management gets that informa- } \\
\text { tion." [Jeff] }\end{array}$ \\
\hline & $\begin{array}{l}\text { Discuss Tools: Converse about creating or } \\
\text { changing dashboards / reports }(13 ; 13)\end{array}$ & $\begin{array}{l}\text { "....why these...mini-charts within this dashboard show up...we've just } \\
\text { made a little chart just for that one ask." [Mandy] }\end{array}$ \\
\hline & Document: Record decisions and history $(1 ; 3)$ & $\begin{array}{l}\text { "I partner with a lot of tourism agencies in different cities I visit... and } \\
\text { they always want to see proof of how much [web] traffic you get." [Milo] }\end{array}$ \\
\hline
\end{tabular}

difference was the prevalence of Monitoring, often overlooked by research on complex analytical work. Data work of dashboard users often involved quick, lightweight activities (Monitor, Summarize, and Lookup goals) to gather information in the course of other work. Note that conversations with data can also be in anticipation of, or part of, conversations through and around data (discussed next).

Conversation through and around data included goals that were primarily communicative. These included discussing data, circulating data artifacts, and producing documentation, and were reported by all interviewees except for Tammy, a self-described "solopreneur". (A second solo entrepreneur, Lisa, reported interactions with her accountant.) Many served as "data intermediaries", making sense of their data and then repackaging and sharing a summary for leadership. Translation is at the core of this role, making data "jump contexts" [17], whether that is temporally for oneself, or across contexts of use or different contextual expectations [18]. Repackaging data into newly created artifacts in this way enabled dashboard users to tell their own story around the data and endow it with additional context and meaning, a concept we call their data voice. Participants from larger organizations also frequently described the 


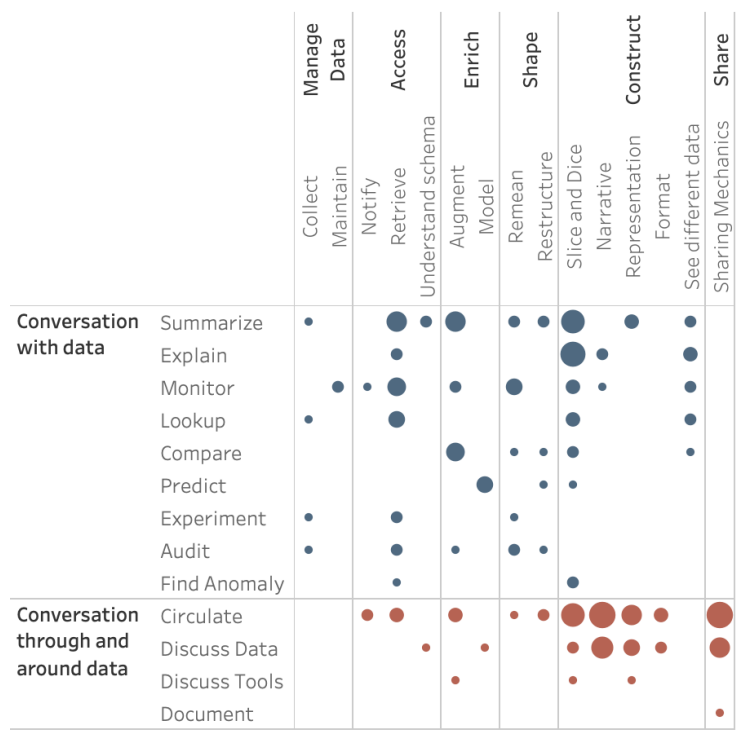

Figure 1. Goals (rows) and tasks (columns) for Dashboard users. Size $=$ number of participants.

pain of getting dashboards or data reports built to support their needs.

While goals represent the high level context of what dashboard users were trying to achieve, breakdown occurred during lower level tasks undertaken to achieve those goals. We organized the tasks into six categories (see Table 3 for details and examples): Manage Data tasks involved collection and maintenance of the raw data, Access tasks involved gaining access to and understanding the data, Enrich tasks focused on adding something, including external data or predictive models, Shape tasks involved restructuring the data or making it more meaningful, Construct tasks involved creating new visual representations and artifacts, and Share tasks involved the mechanics of sharing data and artifacts with others.

As shown in Figure 1, some tasks contributed to many goals while others were more specific. For example, data might be sliced and diced (i.e. grouped, filtered, and aggregated) as part of almost any conversation with, through, or around data, whereas data was only modeled to predict or discuss with others. Similarly, participants only bothered to format if the goal was to circulate or discuss. It is worth highlighting that dashboard users' data tasks went well beyond the "consumption" [Access tasks and Circulation goals] for which dashboards are typically designed. The reality is that dashboard users frequently need to enrich and shape their data, repurpose visualization content, and construct new artifacts and narratives, often in anticipation of conversations with others through and around data.

\section{Case Examples}

Here we introduce four diverse examples. Square brackets reference the goals, tasks, and breakdown mitigation strategies documented in Tables 2 and 3 and Figures 1 and 2. We will use these examples in our subsequent discussion of breakdowns and strategies.

Dustin manages a fitness center at a large aerospace firm. He tracks membership trends and predicts income from membership dues [Goals: Monitor, Predict]. He uses a dashboard to access current and past membership numbers, but for predictive forecasting he uses a spreadsheet [Task: Model; Strategy: Switch Tools], “....I might copy that into say into Excel... Just copy and paste in the fields. And then... I might just make an assumption that it's going to drop because we're coming up on the holidays...So, I'll put a formula in there...let's say in July, decrease by $3 \%$, in August decrease by another 2\%...So, that part of it is manual." This lightweight data modeling task is an example of data enrichment that could not be done in the dashboard. Instead, Dustin had to switch tools, involving an awkward manual transfer of the data and modeling through handwritten formulas. While this manual approach has the advantage of enabling Dustin to encode his expert knowledge, it required substantial rote data manipulation and likely limited the breadth of predictive models to simple linear projections.

Dustin also creates a monthly report for his manager [Goal: Circulate], a process that involves constructing a new artifact from dashboard screenshots, his Excel work, and his interpretation [Tasks: various construction tasks including Narrative]. This again represents a manual process of switching tools and bringing together information from various sources [Strategy: Switch Tools].

Dustin wished his dashboards could allow him to slice and dice data in different ways and enable his what-if analysis [Tasks: Slice and dice, Model]. He saw advantages to doing more of his work within the dashboards and centralizing the data within the database instead of his 
Table 3. Tasks of dashboard users. Numbers indicate how many participants reported (breakdown; the task).

\begin{tabular}{|c|c|c|}
\hline Category & Task and description & Example from our interviews \\
\hline \multirow[t]{2}{*}{ Manage Data } & $\begin{array}{l}\text { Collect: Collect the data needed for anal- } \\
\text { ysis. }(4 ; 5)\end{array}$ & $\begin{array}{l}\text { To calibrate grading practices, several people would periodically grade the same paper; } \\
\text { calibration data was tracked over time. [Amy] }\end{array}$ \\
\hline & $\begin{array}{l}\text { Maintain: Keep the data up to date, which } \\
\text { may include data entry. }(2 ; 2)\end{array}$ & $\begin{array}{l}\text { "....inventory, keeping track of suppliers and vendors... trying to... make that as efficient } \\
\text { as possible in terms of the maintenance of that information." [Tammy] }\end{array}$ \\
\hline \multirow[t]{3}{*}{ Access } & $\begin{array}{l}\text { Notify: Be notified when something hap- } \\
\text { pens, or receive a data push. }(3 ; 3)\end{array}$ & $\begin{array}{l}\text { "We could go weeks before somebody actually looks into this delivery rate, before they } \\
\text { flag that something might be up." [Mandy, requesting anomaly notification] }\end{array}$ \\
\hline & $\begin{array}{l}\text { Retrieve: Gain access to the information } \\
\text { (e.g. by loading a dashboard). }(16 ; 16)\end{array}$ & $\begin{array}{l}\text { "I'm working from a really ugly web-based tool. Literally I sometimes start my morning } \\
\text { with running a report. I let it sit there and spin for like three hours...." [Matthew] }\end{array}$ \\
\hline & $\begin{array}{l}\text { Understand schema: Understand the } \\
\text { data, including its meaning, provenance, } \\
\text { limitations, assumptions. }(5 ; 6)\end{array}$ & $\begin{array}{l}\text { Stacey had to train dashboard users on the relationship between vendors and supplies } \\
\text { and how to link them; confusing because the same item might be procured from several } \\
\text { vendors. [Stacey] }\end{array}$ \\
\hline \multirow[t]{2}{*}{ Enrich } & $\begin{array}{l}\text { Augment: Add new data, including meta- } \\
\text { data. }(12 ; 13)\end{array}$ & $\begin{array}{l}\text { Lisa manually copied data into a spreadsheet to merge credit card sales data from a } \\
\text { Square dashboard with cash sales recorded by hand. [Lisa] }\end{array}$ \\
\hline & $\begin{array}{l}\text { Model: Create a lightweight predictive } \\
\text { model. }(4 ; 4)\end{array}$ & $\begin{array}{l}\text { "What it is not doing is projecting...your sales trend will increase to X over time, or } \\
\text { your category share should be } Y \text { by the time the campaign wraps up. " [Matthew, } \\
\text { describing functionality he wishes he had] }\end{array}$ \\
\hline \multirow[t]{2}{*}{ Shape } & $\begin{array}{l}\text { Remean: Make dimensions and measures } \\
\text { more meaningful (e.g., rename, group, } \\
\text { bin, define metrics). }(9 ; 10)\end{array}$ & $\begin{array}{l}\text { Mandy regularly used a calculator to compute derived metrics on data in dashboards to } \\
\text { make the data more meaningful (e.g., a lift rate computed from raw values). [Mandy] }\end{array}$ \\
\hline & $\begin{array}{l}\text { Restructure: Change the structural orga- } \\
\text { nization of the data. }(6 ; 6)\end{array}$ & $\begin{array}{l}\text { Lisa used a spreadsheet to reorganize her data, "I feel like I'm able to process the } \\
\text { information a little easier, if I organize it exactly the way I want it to." [Lisa] }\end{array}$ \\
\hline \multirow[t]{5}{*}{ Construct } & $\begin{array}{l}\text { Slice and dice: Filter, sort, adjust level of } \\
\text { detail, and pivot to examine different data } \\
\text { slices. }(15 ; 17)\end{array}$ & $\begin{array}{l}\text { "They wanted it in Excel...so they would do pivot tables and look at it one way and } \\
\text { then they'd wanna be able to...look at it by brand instead of by type, like by ring or } \\
\text { bracelet or day...to be able to play with the data..." [Stacey] }\end{array}$ \\
\hline & $\begin{array}{l}\text { Narrative: Add explanatory or contextual } \\
\text { information. }(11 ; 14)\end{array}$ & $\begin{array}{l}\text { "The report is not just an overall report of numbers...They want a narrative about why } \\
\text { the numbers happened." [Jeff] }\end{array}$ \\
\hline & $\begin{array}{l}\text { Representation: Change visual encoding } \\
\text { (including tables). }(8 ; 10)\end{array}$ & $\begin{array}{l}\text { "....somebody else at the company...they would rather see a trend line or something } \\
\text { that's easily digested, whereas I like to be able to really drill down." [Mandy] }\end{array}$ \\
\hline & $\begin{array}{l}\text { Format: Change appearance of the view. } \\
(4 ; 7)\end{array}$ & $\begin{array}{l}\text { "In Keynote...I can remove grid lines...change the formatting of the axis...the colors. I } \\
\text { can make the bars rounded if I wanted to." [Wade, explaining why he switches from } \\
\text { a dashboard to Keynote to create an artifact for sharing] }\end{array}$ \\
\hline & $\begin{array}{l}\text { See different data: See data that is absent } \\
\text { or not easily accessible. }(6 ; 6)\end{array}$ & $\begin{array}{l}\text { Fred often downloaded the data behind a dashboard to see all the data attributes, not } \\
\text { just those visible in the view. [Fred] }\end{array}$ \\
\hline Share & $\begin{array}{l}\text { Sharing Mechanics: Mechanics involved } \\
\text { in communicating with others around } \\
\text { data. }(14 ; 14)\end{array}$ & $\begin{array}{l}\text { "...this could just be my supervisor's preference, she's a bit older. We save it in a } \\
\text { Word document and we send it to her, that what she requests. But we also drop that } \\
\text { same data into a daily template and we use Microsoft Teams." [Amy] }\end{array}$ \\
\hline
\end{tabular}

spreadsheets. However, he could not change the dashboards himself due to lack of time, skill, and tool permissions. Meanwhile, relying on the dashboard builders to make changes was painful [Strategy: Request Service], "It's kind of a pain to get this to change because there's a lot of bureaucracy... and they have other things to do... And unfortunately the [Fitness] center isn't like the biggest priority in an aerospace company."

Kayla is an administrative assistant who synthesizes sales information for senior leadership [Goal: Circulate]. Kayla repeatedly emphasized the importance of curating the data presentation and its aesthetics [Tasks: Format, Representation, Narrative], "....so all of that is just data that we take from CRM or now Salesforce... and we put the lipstick on the pig and put it in pie charts and graphs... and shoot it out." By carefully crafting the data presentation and narrative, Kayla aimed to make the data interpretable and engage her audience. "Make it pretty. I know I keep saying that... it makes people more willing to listen to what everybody on my team has been working on for so long." [Tasks: Representation, Format]
Kayla's workflow involves collecting raw data via email, manually assembling and visualizing it, and then creating a Powerpoint summary that tells a coherent story [Tasks: Collect, Augment, various construction tasks]. She constructs most of her charts manually in Powerpoint, a slow and tedious process [Strategy: Switch Tools]. One of Kayla's great pain points is the inefficiency of validating and merging data emailed to her by over 70 people, a process that occurs repeatedly on a regular schedule. Kayla expressed frustration with her manual workflow. "Obviously, not having to input everything directly myself would be a really nice thing. I've always wished that there was a place where you could input all the data and just hit go... that it wasn't just me clicking away for hours."

Tammy calls herself a "solopreneur", as her online art sales business has exactly one employee, herself. She is most interested in data about online customer behavior and sales, "who's visiting our store, where they're coming from, what are art products that they're looking at....". She monitors web traffic and sales through a 
dashboard that she checks once or twice a day, looking for anything out of the ordinary [Goals: Monitor, Find Anomaly; Task: Retrieve]. One barrier Tammy faces is cross-referencing sales data in the online analytics tool with inventory of art prints in Excel to make sure she maintains sufficient stock [Task: Augment; Strategy: Manual Workaround], "Right now it's...manually looking at both data points and kind of doing my own cross referencing...it's very time consuming and probably not the most efficient....".

Being a solopreneur, Tammy has nobody else to help with analytics. She described her journey of learning how to do more with data despite limited time [Strategy: Learn], “....things kind of fall through the cracks because you're trying to do everything... I finally felt like I was in a place where I could incorporate analytics once I realized okay, the numbers do matter... as opposed to me just kind of guessing...'cause sometimes, the sales don't necessarily represent what people are looking at." Because she had to figure out how to gather the data in the first place [Task: Collect], analytics felt overwhelming at first, "I was spending all these hours just researching all these various different platforms... and I was like, 'Okay, this is too much...I need analytics for my analytics.' I think when I realized it doesn't have to be that detailed...that's when I realized the true value...." [Strategy: Give Up, then later, Learn]. In short, Tammy took a stepwise approach to learning analytics, advancing her data practices only when she hit critical barriers to success.

Miguel is a director of talent acquisition. He tracks diversity and inclusion data, such as employee gender and ethnicity [Goal: Monitor], and screenshares dashboards from his smartphone during a weekly team meeting [Goals: Circulate, Discuss Data; Task: Sharing Mechanics], "We drill down, typically, into the different diversity pools by age, by level of band...." [Task: Slice and dice]. Prior to the meeting, Miguel sends out a Word or Powerpoint summary combining dashboard screenshots with written commentary [Goal: Circulate; Task: Narrative; Strategy: Switch Tools]. Miguel emphasized the importance of sharing more than just the numbers [Task: Narrative], "For me, it's providing a story to the business about where's our current state... and where are we headed ... they're not just looking for stats, they're looking for what sort of story can I tell them?"

Miguel describes himself as a "numbers guy", but still relies on an analyst to create dashboards and prepare screenshots [Strategy: Request Service] because of time pressure, “....she'll never say it, but...I know [analyst name] is probably like, 'Why can't [Miguel] just go in and just do it himself?' Right?...I would like to learn, but I swear to you...It's 5:30 here where I am. And I'm gonna have another two or three hours before I can go home...". His reliance on others plus the real-time nature of meetings means that Miguel sometimes feels pressured by questions he can't answer, "I try not to freak out, but I do at some times, 'cause I don't have the answer on the spot... So I park it, then. I say, 'Hey, well that's a good point. I don't have that data, but I can surely pull it for our next meeting....' " [Goal: Lookup; Task: Slice and dice; Strategy: Postpone and Request Service]. During our conversation, Miguel realized how critical his analyst was to his workflow and decided to train another as backup.

Miguel was quite frustrated by resistance to change, "....the majority of the people...They're just sorta like, 'Well, we'll just wait for [Miguel's] report to come out." Driving change required persistence, "So the journey I've been trying on is just the rinse and repeat. Keep doing it, keep doing it....". A challenge for Miguel was balancing his informal role as analytics champion with his regular job. By demonstrating the value of data, he acquired more responsibilities, training and advising others as well as devising a strategy to scale analytics practices.

\section{Breakdown and Strategies}

As the examples above illustrate, dashboard users had a variety of task needs and their tool ecosystems often failed to serve those needs. We define a breakdown as an instance when a dashboard user could not easily complete their task within their current tool (often a dashboard, but we did not limit our coding of breakdowns to dashboards). Breakdowns could be due to tool functionality, usability, permissions, insufficient knowledge or skill, or lack of time. These moments represent opportunities to innovate better tool support. Below we first discuss strategies employed by dashboard users to escape break- 


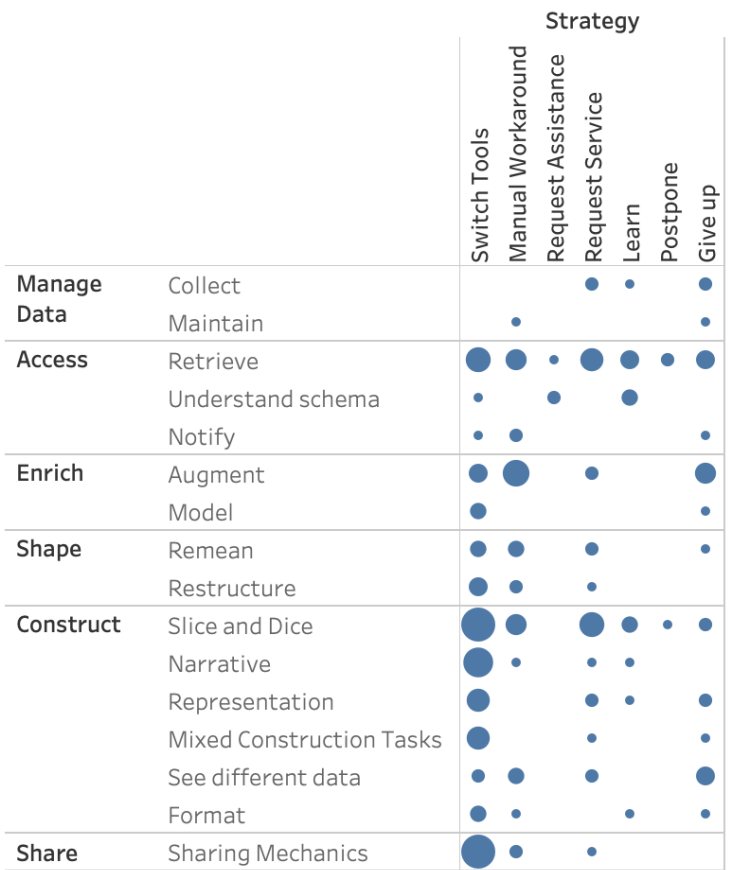

Figure 2. Dashboard users' strategies upon breakdown, grouped by task in which the breakdown occurred. Size $=$ number of participants.

down. We then discuss how analysts employed deliberate strategies to help their dashboard end users avoid breakdown in the first place.

\section{Dashboard Users' Strategies to Escape} Breakdown Figure 2 summarizes strategies that dashboard users adopted to deal with breakdown, and the tasks in which they were employed. The most prevalent strategies involved switching tools, other awkward manual workarounds, requesting that someone else complete the task, or simply giving up.

Switching tools and manual workarounds: Switching tools was a common strategy for various tasks, as shown in Figure 2. Participants took data out of a source artifact and moved it to a new site (usually a spreadsheet, presentation, document, or communication channel) in order to create new meaning or share with others.

By far the most common tool switch was the "data dump": extracting data out of a dashboard for ad hoc analysis in a spreadsheet. This practice was so common that several participants described dashboards deliberately set up as data dumps, often apologizing for 'misusing' their dashboarding tools in this way. "I've created some really ugly Tableau reports just because [dashboard users] can't get the data they want...So they use Tableau as a cross tab generator... so this is all an abuse of Tableau in some ways...And because they're used to looking at walls of Excel data. They're like, 'Okay, this looks good, this looks good...." [Maria, describing a data dump dashboard she constructed for her users]

Data dump activities usually represented breakdown, where completing the task in the dashboard was cumbersome or impossible. In these instances, participants tended to switch to a tool that was both flexible and familiar, the spreadsheet, rather than learning how to modify the dashboard. Another advantage of spreadsheets is their materiality: they allow people to "get their hands on the data". Dashboard users dumped data to spreadsheets for many reasons, encompassing all of the enrichment, shaping, and construction tasks in Table 3 and Figure 2. For example, Dustin dumped data to Excel to predict future gym membership [Model]. Even when starting from clean, curated data in dashboards, dashboard users needed to do a surprising number of data shaping and enrichment tasks in order to answer their questions.

Another common tool switch involved crafting targeted data presentations and surrounding narrative to convey a message, i.e. telling a story with data. Data storytelling is a form of conversing through or around data that puts particular emphasis on constructing a clear and detailed narrative. Data storytelling has become a hot topic in the visualization field, but typically in the context of data journalism aimed at the general public. In contrast, our participants needed to convey a concise, clear, appealing, and convincing message to their management, colleagues, or clients. They didn't need elaborate infographics, but they did need to customize the data presentation and its aesthetic (e.g., simplify to key numbers or a higher level of detail or make it attractive for the audience) and add explanation and recommendations [Construct Tasks]. Despite the seemingly simplistic nature of this work, dashboard users faced many instances of breakdown requiring them to switch tools or employ manual workarounds. Both Kayla and Dustin's stories illustrate the pain of working across tools to craft 
new artifacts. The need to intertwine data with narrative is well recognized as an essential part of both sensemaking and explanation to enable data to jump contexts. Our study highlights the pain dashboard users face in operationalizing their own narratives within their current tool ecosystems.

Reliance on others: As shown in Figure 2, requesting service was a very common strategy, particularly in larger organizations. Dashboard users were much more likely to ask someone else to complete the task for them [Request Service] than to ask for help [Request Assistance] or try to learn new tools or skills [Learn]. Time pressure was often cited as a key reason (e.g., Miguel). Other reasons included lack of skill, role divisions, and lack of access to the right tools. New tools with a lower barrier to entry (e.g., greater flexibility for dashboards to slice and dice data in alternative ways) could help to reduce reliance on others.

Reliance on others was a strategy, but also a burden, because dashboard users had to navigate bureaucracy and long wait times. As Wade put it, "I beg and beg and maybe six months later I might get it...." Dustin's story similarly illustrates this challenge. Meanwhile, analysts felt the burden of having to deal with all the service requests, "You would get inundated by even tedious little not difficult tasks..." [Stacey]

\section{Strategies to Help Dashboard Users Avoid} Breakdown Many analysts were concerned about the low data literacy of their dashboard end users. Literacy issues manifested in our interviews as dashboard users' direct reflection on their limited knowledge, vague or inexpert language (e.g., Dustin referred to the "web guys" and the "data guys"), or limited understanding of their data or tools (e.g., "I don't know what I'm looking at, because now I've got it broken down by municipality and by, oh, now I've got actual just customers...I think this number here, sorry, this Bacct Num, I'm wondering if that's the account number." [Mandy]). To help dashboard users avoid breakdown given these data literacy concerns, analysts adopted deliberate strategies of simplicity, training, and standardization.

Simplicity and training: One strategy was design simplicity and training on how to use dashboards. For instance, "I think just keeping it simple...just the key facts and figures...." [Fred] Analysts were particularly concerned about dashboard users understanding the data schema and its underlying assumptions, meaning that simplicity and clarity of the data design was equally or more important than the visualization design. For example, "....more importantly, we like to be there to make sure that no one misinterprets the data... I mean...they ask a question that either we weren't looking for or that the data does not support." [Brandon].

Data standardization: Another concern was dashboard users involuntarily using inappropriate or unclean data, leading to poor decisions. To avoid these issues, analysts aimed to control the data pipeline and standardize metrics to ensure data quality. As Kaleb put it, "We want to be able to standardize all of our reporting. One of the problems with lots of different people using Excel is...because you can type whatever you want...things can go wrong. I think everyone has heard of some stories... where someone has made an Excel mistake and it's cost the company a lot of money." Stacey had a similar concern, “...data that would be either duplicated...or missing...they were thinking they needed the freshest data so they would just do their own little dumps...just because the tool has thee ability to dump it doesn't mean it's right."

We observed tension between analysts' need for data governance and dashboard users' desire for the freedom and flexibility of spreadsheets. Liz described her organization's data journey as a process of "weaning off Excel". This tension led to the "data dump dashboards" described above, a compromise that enabled access and flexibility for dashboard users while also controlling data quality. The unfortunate cost of this approach for dashboard users is an awkward improvised workflow through a patchwork of ill-fitting tools.

\section{DISCUSSION}

Our results highlight several themes. First, while dashboard users' goals and tasks have overlap with professional analysts, they have different skills, operate in different work contexts, and data may be in service of their goals rather than the focus. Conversations around data (circulation, documentation and discussion) were as critical as conversations with data (including analysis), 
suggesting that tools that focus on supporting analysis alone will fail to support the full scope of how dashboard users need to engage with data. Design for these work contexts needs a new lens that considers the holistic data journey and network of actors.

Second, the linear model of a data analysis pipeline (acquire, wrangle, analyze, report) does not reflect how these workers engage with data, similar to Bigelow's findings [13]. While dashboards were effective tools for quick monitoring and lookup tasks, our participants frequently pulled the data back into tools like Excel to shape and enrich their data and construct new artifacts for their own sensemaking or to converse with others. Spreadsheets have a materiality that offers flexible and hands-on ways to manipulate data. Meanwhile, today's dashboards represent a one-way conversation from the data to the "consumer". Even "interactive" ones offer little room for personal expression. Conversing with others through and around data often requires constructing new artifacts to translate data to new contexts and endow it with new and interpreted meaning. Analytic tools for dashboard users need to support more than simply visualization, including data shaping, enrichment, and construction of new artifacts and narratives. Moving away from dashboard-centered data delivery to more flexible tool ecosystems may be a step towards facilitating data design by dashboard users and shifting data driven organizations to data conversant ones. We share some specific ideas in the next section.

Third, support for data activities are fragmented and poorly integrated into modern data and visualization tools. Dashboards have been quite successful at enabling data documentation and circulation functions within organizations. They also enable some types of data conversations, particularly when the goals are known and the dashboard designer is skilled. However, many conversations with, through, and around data are not easily anticipated. Here dashboard users meet breakdown, relying on help from others or employing awkward workarounds.

We note that dashboard users are a highly diverse group and there are many important factors that may influence their behaviors. Even within our small sample, participants varied widely in terms of demographics, data literacy, tool profi- ciency, educational background, and experience. For example, Lisa, a young artist, was hindered by data literacy, whereas Miguel and Matthew, business executives, had some data training but were severely limited by time.

Moreover, our results can be interpreted within the context of an ongoing journey in which both individuals and organizations may progress towards greater data and analytics proficiency. The learning journeys our participants described were rarely smooth, often requiring an important unmet need to motivate the next step. Tammy and Miguel's stories represent the individual and organizational journeys respectively.

Various aspects of the organizational context influenced the analytics journey, including organization size, bureaucracy, authority, and established work practices. Ingrained work practices and authority perhaps deserve greater attention. While Miguel used his authority to push for change, the reverse was true for Amy, whose older supervisor was an impediment to change. Amy regularly used Excel and Word to manually construct reports, even though the same data was available in a PowerBI dashboard. She expressed frustration with her work process but gave a simple explanation, "I have a supervisor, I just follow what she says to do." Amy started revising her practice only when her supervisor took a two month medical leave. Organizational practices around circulation were particularly difficult to change. For example, participants described taking screenshots of dashboards and putting them into other media. As stated by Maria, "The communication channel is mostly through decks...my hope was that [the end users] would...eventually go to a dashboard. But there is no appetite for that...I think, just culturally it would take a lot of change...." It often took a major breakdown to motivate individual change. Organizational advancement was even more challenging, relying on champions like Miguel with the persistence, authority, and influence to drive change.

\section{OPPORTUNITIES FOR TOOL DESIGN}

Since dashboard users' data work tends to be intermittent, successful tools will need to be designed with low barriers to entry so they are easy to pick up and use. Reducing the tedious manual effort of moving data from one platform 
to another would, in itself, greatly facilitate the activities of dashboard users. Such tool integrations could bring data access to environments that dashboard users already occupy, such as messaging platforms.

Another promising approach is to re-envision dashboard tools so they can support unanticipated use cases, transforming them into interactive data experiences. This could include flexible slicing and dicing mechanisms, natural language interaction to enable users to ask new questions about the underlying data, and machine learning tools that automatically surface relationships or anomalies. Ideally these interactive data experiences should be curated by an analyst (in the way dashboards are already curated data portals) so they are customized for particular users and data. As such, we will also need novel authoring tools to support analysts in curating these data experiences.

For dashboard users to organize their own content or restructure it for conversations with others, new mashup tools are worth exploring. Imagine if a dashboard user could easily snag content from different dashboards, arrange it in a new layout, customize the formatting, add new narratives to construct their own data story, and share their new data artifact with others, all without leaving the dashboarding ecosystem.

Most visualization and dashboard tools require users to work with aggregated data and data abstractions (e.g. fields "Name" and "height"), whereas spreadsheets support seeing and directly manipulating data values (e.g. "Jane Smith", "152 cm"). As working with abstractions can be challenging for nonexperts, particularly when creating data transformations and calculations, tools that enable people to more directly see and manipulate data values and records may be promising. Can future research bring some of the direct data manipulation affordances of spreadsheets into dashboards and advanced analytics tools? Such embodied and hands-on interaction are gaining recent popularity in the visualization literature; examples include physical data construction [20] and multimodal interaction (e.g., [21]). Further, the translation work between vague questions and machine-understandable questions is challenging for many dashboard users. Building cooperative, machine-assisted ways to guide disambiguation and refinement of analytic questions could provide valuable support regardless of the interaction modality. We encourage future work that builds on these directions.

\section{LIMITATIONS}

Our research used a qualitative approach to understand the practices, barriers, goals, and tasks of dashboard users. We chose this approach to capture the diversity of dashboard users' experiences, but the approach also has limitations. Interviewees self-selected to participate and as such we do not capture the perspectives of all dashboard users. Notably, individuals who experience such significant barriers that they forego dashboard use are absent from our study, but may still be relevant to consider. Where we aimed for breadth, future studies could also drill into depth with users of specific dashboard platforms or within specific domains. Our study focused on barriers, as these arose organically from the interview data. We argue that the choice of barriers allowed us to identify actionable targets for future research, as the general sentiment was that dashboard users were not well supported by the current software tooling. To mitigate these limitations we transparently report on our methodological approach and qualitative codes.

For clarity, we characterized dashboard users and analysts as two separate groups, but the reality is more nuanced. Our research illuminated the journeys taken by some of our participants towards greater data and analytics literacy. Some dashboard users will take this journey far enough to become analysts. Similarly, some analysts may become dashboard users when they move into leadership roles - where they employ analysts rather than doing that work themselves. We encourage future work to better understand these journeys and transformations.

\section{CONCLUSION}

Data and visualization are not just for analysts. Data democratization means that a much broader population is actively engaging in data conversations, often through dashboards. Yet dashboard users are employing improvised, awkward workflows because their needs are not well supported. Core to the problem is that dashboard use is not just "consumption" or "analysis", nor 
is analysis a distinct, separate activity. Dashboard users are collecting, shaping, and enriching their data. They're transforming the data to give it new meaning and then translating it to new contexts by constructing and sharing new artifacts. Do we expect them to use tools designed for circulation to support these rich conversational goals? Do we expect them all to gain the skills of professionals before they can use analytics tools? Or can we design tools that meet them where they are?

As a data visualization community, our aim is to support people in their conversations with, through, and around data. But supporting truly fluent data conversations means that we need to first understand the complex data work of everyone who uses data, not just analysts, and then build easy to use but powerful tools that interoperate seamlessly and support these holistic workflows. A broad and diverse population of people use data every data to make decisions that influence our world. Making their data work easier represents an enormous opportunity for impact of visual analytics tools and processes. Please join us in embracing dashboard users. Together, let's design the tools that help them find their data voice.

\section{ACKNOWLEDGMENT}

We thank Michelle Kosterich and Jeff Pettiross for their assistance with interviews and Matthew Brehmer for feedback on the draft.

\section{- REFERENCES}

1. J. Liu, N. Boukhelifa, and J. R. Eagan, "Understanding the Role of Alternatives in Data Analysis Practices," IEEE Trans. Visualization and Computer Graphics, vol. 26, no. 1, pp. 66-76, Jan. 2020.

2. A. Sarikaya, M. Correll, L. Bartram, M. Tory, and D. Fisher, "What do we talk about when we talk about dashboards?" IEEE Trans. Visualization and Computer Graphics, vol. 25, no. 1, pp. 682-692.

3. E. Kandogan, A. Balakrishnan, E. M. Haber, and J. S. Pierce, "From data to insight: Work practices of analysts in the enterprise," IEEE Computer Graphics and Applications, vol. 34, no. 5, pp. 42-50.

4. S. Kandel, A. Paepcke, J. M. Hellerstein, and J. Heer, "Enterprise data analysis and visualization: An interview study," IEEE Trans. Visualization and Computer Graphics, vol. 18, no. 12, pp. 2917-2926.
5. S. Alspaugh, N. Zokaei, A. Liu, C. Jin, and M. A. Hearst, "Futzing and moseying: Interviews with professional data analysts on exploration practices," IEEE Trans. Visualization and Computer Graphics, vol. 25, no. 1, pp. 22-31.

6. M. Muller, I. Lange, D. Wang, D. Piorkowski, J. Tsay, Q. V. Liao, C. Dugan, and T. Erickson, "How data science workers work with data: Discovery, capture, curation, design, creation," in Proc. CHI Conf. Human Factors in Computing Systems. ACM, 2019, pp. 115. [Online]. Available: http://doi.org/10.1145/3290605. 3300356

7. K. E. Weick, Sensemaking in organizations, ser. Foundations for organizational science. Sage Publications, 1995.

8. N. Boukhelifa, M.-E. Perrin, S. Huron, and J. Eagan, "How data workers cope with uncertainty: A task characterisation study," in Proc. CHI Conf. Human Factors in Computing Systems. ACM, 2017. [Online]. Available: http://doi.org/10.1145/3025453.3025738

9. L. Koesten, E. Kacprzak, J. Tennison, and E. Simperl, "Collaborative practices with structured data: Do tools support what users need?" in Proc. Conf. Human Factors in Computing Systems, 2019, pp. 1-14.

10. G. Convertino and A. Echenique, "Self-service data preparation and analysis by business users: New needs, skills, and tools," in Proc. CHI Conf. Extended Abstracts on Human Factors in Computing Systems, ser. CHI EA '17. ACM, pp. 1075-1083. [Online]. Available: https://doi.org/10.1145/3027063.3053359

11. S. Erete, E. Ryou, G. Smith, K. M. Fassett, and S. Duda, "Storytelling with data: Examining the use of data by non-profit organizations," in Proc. ACM Conf. Computer-Supported Cooperative Work \& Social Computing, 2016, pp. 1273-1283.

12. L. Bartram, M. Correll, and M. Tory, "Untidy data: The unreasonable effectiveness of tables," arXiv preprint arXiv:2106.15005, 2021.

13. A. Bigelow, S. Drucker, D. Fisher, and M. Meyer, "Reflections on how designers design with data," in Proc. 2014 International Working Conf. Advanced Visual Interfaces, 2014, pp. 17-24.

14. E. Dimara, H. Zhang, M. Tory, and S. Franconeri, "The unmet data visualization needs of decision makers within organizations," IEEE Transactions on Visualization and Computer Graphics, 2021.

15. G. Neff, B. Fiore-Silfvast, and C. S. Dossick, Materiality: Challenges to Communication Theory, ser. International Communication Association Theme Book 2013: 
Challenging Communication Research. Peter Lang, 2014.

16. A. Tanweer, B. Fiore-Gartland, and C. Aragon, "Impediment to insight to innovation: understanding data assemblages through the breakdown-repair process," Information, Communication \& Society, vol. 19, no. 6, pp. 736-752. [Online]. Available: http://www.tandfonline. com/doi/full/10.1080/1369118X.2016.1153125

17. G. J. Downey, Making Media Work. The MIT Press, Feb 2014, p. 141-166. [Online]. Available: http://mitpress.universitypressscholarship. com/view/10.7551/mitpress/9780262525374.001.0001/ upso-9780262525374-chapter-8

18. B. Fiore-Gartland and G. Neff, "Communication, mediation, and the expectations of data: Data valences across health and wellness communities," Intl. J. Communication, vol. 9, no. 1, pp. 1466-1484, 2015. [Online]. Available: https://ijoc.org/index.php/ijoc/article/ view/2830

19. K. Charmaz, Constructing Grounded Theory, ser. Introducing Qualitative Methods series. SAGE Publications, 2014. [Online]. Available: https://uk.sagepub.com/ en-gb/eur/constructing-grounded-theory/book235960

20. S. Huron, Y. Jansen, and S. Carpendale, "Constructing visual representations: Investigating the use of tangible tokens," IEEE Trans. Visualization and Computer Graphics, vol. 20, no. 12, pp. 2102-2111, 2014.

21. A. Srinivasan, B. Lee, and J. T. Stasko, "Interweaving multimodal interaction with flexible unit visualizations for data exploration," IEEE Transactions on Visualization and Computer Graphics, 2020.

Melanie Tory is Director of Data Visualization Research at the Roux Institute of Northeastern University, Portland, ME, USA. She received her Ph.D. from Simon Fraser University in 2004. Tory has broad research interests in visualization and human-data interaction and focuses on empowering less experienced users to engage with data. Tory is Associate Editor of IEEE Computer Graphics and Applications, IEEE Transactions on Visualization and Computer Graphics, and Computer Graphics Forum. She is a member of IEEE and the IEEE Computer Society. Contact her at m.tory@northeastern.edu.

Lyn Bartram is Professor of Interactive Arts + Technology and Director of the Vancouver Institute of Visual Analytics at Simon Fraser University, Surrey, BC, Canada. She received the Ph.D. in 2001 from Simon Fraser University. Her interdisciplinary research combines computer science, perceptual and cognitive science, and social science to explore how people understand and interact with data. She has an extensive background in training and research with data users in visualization and visual analytics. She is a member of IEEE. Contact her at lyn@sfu.ca.

Brittany Fiore-Gartland is a Director of User Research at Tableau, Seattle, USA. She received her Ph.D. in 2014 from the University of Washington. Fiore-Gartland is a multidisciplinary researcher who brings an ethnographic and sociological lens to the design of data intensive technologies in organizations and the changing nature of data work. Her research has focused on how groups collaborate and communicate with data, human-centered machine learning, and data ethics. Contact her at bfioregartland@tableau.com.

Anamaria Crisan is a Senior Research Scientist at Tableau, Seattle, USA. She received her Ph.D. in 2019 from the University of British Columbia. Crisan is a multidisciplinary researcher who integrates techniques and methods from applied statistics, machine learning, human computer interaction, and data visualization. Her research focuses on the intersection of Data Science and Data Visualization, specifically how humans can collaboratively work together with $\mathrm{ML} / \mathrm{Al}$ systems through visual interfaces. Contact her at acrisan@tableau.com. 